\title{
DEVELOPMENT OF METHODS FOR DETERMINING THE COORDINATES OF FIRING POSITIONS OF ROVING MORTARS BY A NETWORK OF COUNTER-BATTERY RADARS
}

\author{
Hennadii Khudov \\ Department of Radar Troops Tactic ${ }^{1}$ \\ 2345kh_hg@ukr.net \\ Iryna Yuzova \\ Department of Information Technologies \\ Civil Aviation Institute
}

228 Klochkivska str., Kharkiv, Ukraine, 61023

uzik25@ukr.net

Bohdan Lisohorskyi

Department of Radar Troops Tactic ${ }^{1}$

Yuriy Solomonenko

Department of Radar Troops Tactic ${ }^{1}$

solom_169@ukr.net

\section{Serhii Mykus}

Department of Information Technologies and Information Security Employment ${ }^{2}$ serg.mikus@gmail.com

\section{Artem Irkha}

Department of Space Systems and Geographic Information Support ${ }^{2}$

art_irkha@ukr.net

\section{Volodymyr Onishchuk}

634 Head Office Military Missions

Ministry of Defence of Ukraine

2 Yuri Illenko str., Kyiv, Ukraine, 04050

onihcuk181@i.ua

\section{Serhii Sukonko}

Department of Tactical and Special Training

National Academy of the National Guard of Ukraine

3 Zakhysnykiv Ukrainy sq., Kharkiv, Ukraine, 61001

sukonko_sergei@ukr.net

Galina Semiv

Department of Rocket Artillery Armament ${ }^{3}$

Semen Bondarenko

Department of Rocket Artillery Armament ${ }^{3}$

${ }^{1}$ Ivan Kozhedub Kharkiv National Air Force University

77/79 Sums'ka str., Kharkiv, Ukraine, 61023

\footnotetext{
${ }^{2}$ National Defence University of Ukraine named after Ivan Cherniakhovskyi 28 Povitroflotskiy ave., Kyiv, Ukraine, 03049

${ }^{3}$ Hetman Petro Sahaidachnyi National Army Academy 32 Heroiv Maidanu str., Lviv, Ukraine, 79026
} 


\begin{abstract}
The mathematical formulation of the problem of determining the coordinates of targets in the network of counter-battery radars is formulated. It has been established that the problem of estimating the coordinates of targets in the network of counter-battery radars for an excessive number of estimates of primary coordinates should be considered as a statistical problem. The method for determining the coordinates of the firing positions of roving mortars has been improved, in which, in contrast to the known ones, the coordinates of targets on the flight trajectory are coordinated with space and time and the information is processed by a network of counter-battery radars. The developed simulation mathematical model for determining the coordinates of the firing positions of roving mortars by a network of counter-battery radars. Simulation modeling of the method for determining the coordinates of the firing positions of roving mortars by a network of counter-battery radars has been carried out. It has been established that the use of a network of radars makes it possible to increase the accuracy of determining the coordinates of the firing means on average from $23 \%$ to $71 \%$, depending on the number of counter-battery radars in the network. It has also been found that the appropriate number of counter-battery warfare radars in the network is three or four. A further increase in the number of counter-battery warfare radars in the network does not lead to a significant increase in the accuracy of determining the coordinates of artillery and mortar firing positions. In carrying out further research, it is necessary to develop a method for the spatial separation of elements of a group of targets and interfering objects by a network of counter-battery warfare radars.
\end{abstract}

Keywords: method of determining coordinates, firing position, roving mortar, radar, counter-battery warfare.

DOI: $10.21303 / 2461-4262.2021 .001821$

\title{
1. Introduction
}

The most common types of terrorist attacks are grenade launcher attacks and mortar attacks. The use of roving mortars by terrorists is due to the following:

- firstly, mortars are quite easy to make in a handicraft way from scrap materials;

- secondly, terrorists often locate mortar firing positions in residential areas, refugee camps, near schools, hospitals, hiding behind a «human shield»;

- thirdly, regular shelling from mortars has a fairly strong psychological impact.

The experience of conducting counter-battery activities (CBA) showed a number of problems in countering roving mortars. As a means of counter-battery warfare, let's consider CBA radars AN/TPQ-48A (USA) (and a modification of AN/TPQ-49). One of the main problems is significant errors in determining the position of the mine and, accordingly, the coordinates of the firing positions of roving mortars to deliver an adequate retaliatory strike in order to destroy the firing weapon. Improving the accuracy of determining the coordinates of the firing positions of roving mortars is especially important when firing from residential areas, school grounds, kindergartens, etc.

The existing methods for determining the position of the mine and, accordingly, the coordinates of the firing positions of roving mortars provide for the autonomous operation of several CBA radars. In this case, information from each CBA radar is processed separately, regardless of the operation of another CBA radar.

It is proposed to combine autonomous CBA radars into a network. When building a CBA radars network, a number of additional opportunities arise associated with the use of multi-radar or multi-position methods for processing radar information:

- increasing the probability of detecting targets at a given time interval by the CBA radars network in comparison with an autonomous radar;

- increasing the likelihood of detecting targets by irradiating them from different angles by spatially spaced CBA radars;

- reducing the time of setting up and improving the quality of tracking target trajectories by increasing the rate of information update in the CBA radars network in comparison with an autonomous radar;

- improving the accuracy of determining the coordinates of targets by averaging or weighting data integration in the CBA radars network;

- increasing the accuracy of determining the coordinates of targets through the use of multi-position methods for determining the coordinates of targets.

So, it is relevant to develop a method for determining the coordinates of the firing positions of roving mortars by the CBA radars network. 
The aim of the article is to improve the accuracy of determining the coordinates of the firing positions of roving mortars through the use of the CBA radars network.

To achieve the aim, the following objectives have been set:

- to develop a method for determining the coordinates of the firing positions of roving mortars;

- to carry out simulation modeling of the method for determining the coordinates of the firing positions of roving mortars by the CBA radars network;

- to determine the appropriate number of CBA radars in the network.

The experience of the advanced countries of the world shows that the Counter-Rockets, Artillery and Mortar (C-RAM) concept can be used as a response to mortar attacks, violations of international humanitarian law and the infliction of unintentional civilian casualties [1]. C-RAM is a set of measures to counter missile, artillery and mortar attacks [1]. For example, in Iraq (2004), a working group was created to study C-RAM issues from the Training and Doctrine Command (TRADOC) of the US Army [2]. The result of the group's work was the creation of a consolidated tactical unit, which combined the forces and means of anti-aircraft artillery air defense of the ground forces, field artillery of the ground forces and air defense of the United States [2, 3]. The main component of such a unit is the AN/TPQ-48A CBA radar (and the AN/TPQ-49 modification).

The measurements of the object coordinates of the CBA radars are carried out in a spherical coordinate system and include measurements of the radial range, angular coordinates (azimuth and elevation) and radial velocity of the target $[4,5]$. Most often, the direct radar method is used to measure the range, based on measuring the delay time of the reflected signal relative to the probing one $[4,5]$. With the amplitude method of measuring the range, the lag time of the characteristic change in the amplitude of the received radar signal is determined. The most common modulation of rangefinder radars is pulse-amplitude modulation $[4,5]$. Measurements can be carried out both sequentially in time (by scanning the antenna pattern) or by instantaneous comparison of signals (monopulse method) [6]. The main disadvantage of the known methods of measuring coordinates by existing CBA radars is the low accuracy of determining the coordinates of the mine (target) and, accordingly, the coordinates of the firing positions of roving mortars [4-6].

One of the possible ways to improve the accuracy of determining the coordinates of objects in radar systems is the use of multi-position radar systems [7, 8]. At the same time, system effects are used when combining autonomous radars into synchronous multiradar systems (MRS) with the implementation of coherence of varying degrees and compatible echo reception [9]. The key issue that determines the possibility of creating a synchronous-coherent MRS of space-separated radars is the need to fulfill the conditions $[8,9]$ : frequency synchronization, phase synchronization, time synchronization, coordinated airspace survey.

In [10], a method is proposed for determining the coordinates of the motion parameters of an object with nonlinear motion using only angular information. The comparison of the accuracy of determining the coordinates and parameters of the object's motion by the methods of N-bearings and N-polynomials is carried out. It has been established that the method [10] is effective for uniform rectilinear, uniformly accelerated and uniform movements of an object and can be used to determine the parameters of movement of objects in the CBA radars.

In [11], a method for determining the location of an object by the Saibel direction finder is proposed. In [12], an algorithm is proposed for determining the coordinates of radio emission sources from an aircraft based on phase-time signal information from two receiving modules. However, the methods $[11,12]$ are effective only for determining the coordinates of objects in passive systems. Also, the definitions of the coordinates of objects in passive systems are considered in the review work [13].

In [9], a variant of constructing an MRS and methods for increasing the efficiency of radar detection of subtle air objects are proposed. However, the methods [9] provide for the MRS construction as part of a radar with mechanical rotation.

Methods of statistical synthesis and analysis of devices for detecting and measuring the coordinates of objects in multi-position radio engineering systems are considered in [14]. However, in [14], the features of target detection in the CBA radars were not considered.

The fundamentals of the theory of reception and processing of signals in MRS are developed in [8]. The peculiarity of these systems is the use, to one degree or another, of spatio-temporal 
methods of information processing. This information is embedded in wave fields and radio signals, which are received simultaneously at spatially separated receiving points (including mobile ones). In this case, both active and passive methods of radio reception are used. In [8], a statistical theory of detection, determination of object coordinates, determination of principles of interposition identification of single measurements and target trajectories based on the use of parametric and coordinate gates in multiradar active and passive systems is presented. However, in [8], the features of detecting objects by the CBA radars network are not considered.

Further development of radar systems is carried out in the direction of combining separate surveillance radars with mechanical rotation in a small base MRS with joint processing of radar information $[15,16]$. A variant of organizing joint information processing in an active-passive radar system is considered to increase the probability of detecting an airborne object and joint processing of coordinate information to improve the accuracy of determining coordinates.

In [17, 18], the principles of construction and options for the practical implementation of spaced-apart radar systems are considered. Expressions are obtained for assessing the potential capabilities of spaced radar systems to identify and determine the coordinates of airborne objects, the dependences of the characteristics of spaced radar systems on the number of radars are given, and is used to illuminate an airborne object. However, in [17, 18], the CBA radars integration in order to improve the quality of object detection is not considered.

For further research in order to improve the accuracy of determining the coordinates of the firing positions of roving mortars, let's set the task of developing a method for determining the coordinates of the firing positions of roving mortars by using the CBA radars network.

\section{Materials and methods}

The research used methods of system analysis, methods of probability theory and mathematical statistics, methods of statistical radio engineering, mathematical apparatus of matrix theory, methods of differential calculus, methods of mathematical modeling, methods of radar theory, methods of multiposition radar, methods of optimization theory; iterative methods, methods of mathematical modeling. During the experimental studies, the method of statistical tests (Monte Carlo method) was used. When validating the proposed solutions, analytical and empirical methods of comparative research were used.

The following constraints and assumptions were adopted during modeling:

- CBA radar AN/TPQ-49 are considered as a means of radar;

- conditions for ensuring a coordinated view of the airspace by the CBA radars network have been fulfilled;

- conditions for the time synchronization of the operation of the CBA radars in the quality network have been met;

- roving mortars of $82 \mathrm{~mm}$ and $120 \mathrm{~mm}$ calibers are considered as firing means;

- mines of $82 \mathrm{~mm}$ and $120 \mathrm{~mm}$ calibers are considered as targets in the CBA radars;

- there is one target within the coverage area of the CBA radars network;

- corrections for meteorological conditions during shooting were taken into account (the state of the atmosphere in the plane of shooting (wind, pressure, air temperature));

- trajectory of the target (exchange) is taken as ballistic.

\section{Research and discussion}

The location of the firing position of the roving mortar (hereinafter referred to as the firing position) is determined by the vector $\zeta\left(x_{A}, y_{A}, z_{A}\right)$, where $x_{A}, y_{A}, z_{A}$ - the coordinates of point $A$ of the firing position. The mine, flying out of the firing means, is the target for the CBA radar. Let's consider the CBA radars network with $N \geq 2$ CBA radar single-position, in which the coordinates of the target are determined by forming the resulting single measurement. Within the coverage area of the network, each $n$-the CBA radars conducts $I$ primary measurements of azimuth $\tilde{\alpha}_{i n}$, elevation $\tilde{\beta}_{i n}$ and range $\tilde{D}_{i n}$ (the symbol « » means the estimate of the corresponding coordinate) $(i=1 \ldots I, n=1 \ldots N)$.

Within the coverage area of the network, all CBA radars measure the primary coordinates of the target simultaneously. 
By the characteristic of the target at each moment of time, let's define the state vector $X^{T}=(\alpha, \beta, D)$, which contains three coordinates of the target (azimuth, elevation and range, respectively). To determine the coordinates of the firing position of a roving mortar, it is necessary to determine the spatial coordinates of the target (mines). To determine the spatial coordinates of the target, it is necessary to have at least three independent measurements of the primary coordinates. The measurements of the primary coordinates of the target by the radar network can be summarized in an observation matrix $\tilde{U}(\tilde{\alpha}, \tilde{\beta}, \tilde{D})$ (expression (1)) of size $X^{T}=(\alpha, \beta, D)$ where I is the number of measurements of the coordinates of the target of each CBA radar.

$$
\tilde{U}(\tilde{\alpha}, \tilde{\beta}, \tilde{D})=\left|\begin{array}{cccc}
\tilde{\alpha}_{11} & \tilde{\alpha}_{12} & \ldots & \tilde{\alpha}_{1 I} \\
\tilde{\beta}_{11} & \tilde{\beta}_{12} & \ldots & \tilde{\beta}_{11} \\
\tilde{D}_{11} & \tilde{D}_{12} & \ldots & \tilde{D}_{11} \\
\tilde{\alpha}_{21} & \tilde{\alpha}_{22} & \ldots & \tilde{\alpha}_{2 I} \\
\tilde{\beta}_{21} & \tilde{\beta}_{22} & \ldots & \tilde{\beta}_{2 I} \\
\tilde{D}_{21} & \tilde{D}_{22} & \ldots & \tilde{D}_{2 I} \\
\ldots & \ldots & \ldots & \ldots \\
\tilde{\alpha}_{N 1} & \tilde{\alpha}_{N 2} & \ldots & \tilde{\alpha}_{N I} \\
\tilde{\beta}_{N 1} & \tilde{\beta}_{N 2} & \ldots & \tilde{\beta}_{N I} \\
\tilde{D}_{N 1} & \tilde{D}_{N 2} & \ldots & \tilde{D}_{N I}
\end{array}\right| .
$$

From expression (1) it is seen that when using the CBA radars network, there are redundant measurements that can be used to increase the accuracy of the resulting unit measurement, eliminate uncertainty and interpositional identification of targets. Let's suppose that, in accordance with the geometric configuration of the CBA radars network, the measurement vector of the primary coordinates of the target $\tilde{U}(\tilde{\alpha}, \tilde{\beta}, \tilde{D})$ is related to the state vector $X^{T}=(\alpha, \beta, D)$ by the following general functional dependence (2):

$$
\tilde{U}(\tilde{\alpha}, \tilde{\beta}, \tilde{D})=h(X(\alpha, \beta, D)),
$$

where $h^{T}(X)=\left[h_{1}(\mathrm{X}), \ldots, h_{k}(\mathrm{X})\right]$ - vector of known functions that determine the dependence of the primary coordinates of the target on the components of the state vector $X^{T}=(\alpha, \beta, D)$.

Estimates of azimuth $\tilde{\alpha}$, elevation $\tilde{\beta}$ and range $D$ in expression (2) differ from the true values of azimuth $\alpha$, elevation $\beta$ and range $D$ by the amount of measurement errors.

Each of the equations (2) with fixed values of the left sides determines the surface of the target position in space. By measuring the primary coordinates, the azimuth and range correspond to the surface of the position in the form of a vertical plane through the CBA radars and the target and the sphere centered at the CBA radars, respectively. The coordinates of the point of intersection of at least three of the specified surfaces correspond to the spatial coordinates of the target. Thus, the task of determining the coordinates of targets in the CBA radars network is to determine, in one way or another, the coordinates of the point of intersection of the position surfaces and the formation of the resulting single measurement in the coordinate system of the CBA radars network. Mathematically, this problem is equivalent to finding the roots of a system of $N$ equations (2) and can be solved in various ways.

Expression (2) takes into account the fact that when measuring the signal parameters, various kinds of errors arise. This leads to errors in measuring the primary coordinates of the target, and hence the corresponding surfaces of the position. Therefore, in fact, only estimates of the primary coordinates of the target $\tilde{X}^{T}=(\tilde{\alpha}, \tilde{\beta}, \tilde{D})$ can be obtained, which differ from the true values $X^{T}=(\alpha, \beta, D)$. At each moment of time, to obtain an estimate of the state vector $\tilde{X}^{T}=(\tilde{\alpha}, \tilde{\beta}, \tilde{D})$, $(3 \times N)$ measurements of each coordinate are carried out. The observation matrix at an arbitrary $k$-th moment in time ( $k$-th dimension) can be represented as a column vector (expression (3)):

$$
\tilde{U}_{k}(\tilde{\alpha}, \tilde{\beta}, \tilde{D})=\left(\begin{array}{llllllllll}
\tilde{\alpha}_{1 k} & \tilde{\beta}_{1 k} & \tilde{D}_{1 k} & \tilde{\alpha}_{2 k} & \tilde{\beta}_{2 k} & \tilde{D}_{2 k} & \ldots & \tilde{\alpha}_{N k} & \tilde{\beta}_{N k} & \tilde{D}_{N k}
\end{array}\right)^{T} .
$$


In the general case, the functional dependencies connecting the estimates of the spatial coordinates of the target $\tilde{X}^{T}=(\tilde{\alpha}, \tilde{\beta}, \tilde{D})$ with the primary $\tilde{U}(\tilde{\alpha}, \tilde{\beta}, \tilde{D})$ have the form (4):

$$
\left\{\begin{array}{l}
\tilde{D}=f_{D}\left[\tilde{U}_{1}(\tilde{\alpha}, \tilde{\beta}, \tilde{D}), \ldots, \tilde{U}_{N}(\tilde{\alpha}, \tilde{\beta}, \tilde{D})\right], \\
\tilde{\alpha}=f_{\alpha}\left[\tilde{U}_{1}(\tilde{\alpha}, \tilde{\beta}, \tilde{D}), \ldots, \tilde{U}_{N}(\tilde{\alpha}, \tilde{\beta}, \tilde{D})\right], \\
\tilde{\beta}=f_{\beta}\left[\tilde{U}_{1}(\tilde{\alpha}, \tilde{\beta}, \tilde{D}), \ldots, \tilde{U}_{N}(\tilde{\alpha}, \tilde{\beta}, \tilde{D})\right],
\end{array}\right.
$$

where

$$
f_{D}\left[\tilde{U}_{1}(\tilde{\alpha}, \tilde{\beta}, \tilde{D}), \ldots, \tilde{U}_{N}(\tilde{\alpha}, \tilde{\beta}, \tilde{D})\right], f_{\alpha}\left[\tilde{U}_{1}(\tilde{\alpha}, \tilde{\beta}, \tilde{D}), \ldots, \tilde{U}_{N}(\tilde{\alpha}, \tilde{\beta}, \tilde{D})\right], f_{\beta}\left[\tilde{U}_{1}(\tilde{\alpha}, \tilde{\beta}, \tilde{D}), \ldots, \tilde{U}_{N}(\tilde{\alpha}, \tilde{\beta}, \tilde{D})\right]
$$

- functions obtained by direct solution of the inverse algebraic equation $\tilde{X}(\tilde{\alpha}, \tilde{\beta}, \tilde{D})=$ $=\arg h[\tilde{U}(\tilde{\alpha}, \tilde{\beta}, \tilde{D})]=f[\tilde{U}(\tilde{\alpha}, \tilde{\beta}, \tilde{D})]$ formed from expression $(2) ; \tilde{U}_{1}(\tilde{\alpha}, \tilde{\beta}, \tilde{D}), \ldots, \tilde{U}_{N}(\tilde{\alpha}, \tilde{\beta}, \tilde{D})-$ primary measurements of the first, second, ..., $n$ radars of the CBA radars network.

A feature of solving CBA problems is that the end result is not the determination of the coordinates of the target, but the determination of the coordinates of the firing position and the point of the mine fall. Therefore, along with the vector of primary observations $\tilde{U}(\tilde{\alpha}, \tilde{\beta}, \tilde{D})$, the state vector $\tilde{X}^{T}=(\tilde{\alpha}, \tilde{\beta}, \tilde{D})$, let's consider the vector of trajectory measurements $\tilde{\xi}=(\tilde{x}(t), \tilde{y}(t), \tilde{z}(t))^{T}-$ the vector of estimates of the coordinates of the target positions on the flight trajectory, obtained as a result of processing the primary measurements at a certain moment in time. These estimates of the vector of trajectory measurements $\tilde{\xi}=(\tilde{x}(t), \tilde{y}(t), \tilde{z}(t))^{T}$ are determined in a Cartesian rectangular coordinate system as $\tilde{x}\left(t_{i}\right), \tilde{y}\left(t_{i}\right), \tilde{z}\left(t_{i}\right), i=1 \ldots I$. Primary measurements of target coordinates contain errors of primary measurements of azimuth $\partial \alpha_{n i}$, elevation $\partial \beta_{n i}$, range $\partial D_{n i}$ of the $n$-th radar of the CBA on the $i$-th dimension. Let's designate $\Delta_{U i}=\left(\partial \alpha_{1 i}, \partial \beta_{1 i}, \partial D_{1 i}, \ldots \partial \alpha_{N i}, \partial \beta_{N i}, \partial D_{N i}\right)^{T}-$ the vector of errors on the $i$-th primary measurement of each CBA radar; $\alpha_{n i}, \beta_{n i}, D_{n i}-$ «exact» values of azimuth, elevation and range, respectively, measured by the $n$-th CBA radar at the $i$-th measurement. These «exact» values can be reduced to a matrix $U(\alpha, \beta, D)$ of dimension $(3 \times N) \times I$. Since the primary measurements contain an error, the estimates of the vector of trajectory measurements $\tilde{\xi}=(\tilde{x}(t), \tilde{y}(t), \tilde{z}(t))^{T}$ of the coordinates of the target on the $i$-th dimension will also contain errors in determining the coordinates of the target $\partial x_{i}, \partial y_{i}, \partial z_{i}$, caused by the presence of errors in the primary measurements. Let's denote $x\left(t_{i}\right), y\left(t_{i}\right), z\left(t_{i}\right)$ - «exact» values of the target coordinates at time $t_{i}$ (on the $i$-th dimension); $\Delta_{\xi i}=\left(\partial x_{i}, \partial y_{i}, \partial z_{i}\right)^{T}$ - the vector of errors in determining the coordinates of the target on the $i$-th dimension. Let's pose the problem of finding the coordinates of the target (vector of trajectory measurements) in the CBA radars network. It is expedient to consider such a problem of estimation by an excessive number of estimates of primary coordinates as a solution to some statistical problem. Let's assume that at point $C_{i}$ of the trajectory with unknown coordinates $x_{i}, y_{i}, z_{i}$ at time $t_{i}$ there is a target (mine). At $N$ receiving points (CBA radars network) at a given time, azimuths, elevation angles and slant ranges to point $\mathrm{C}$ are measured. As a result, an observation vector of dimension $(3 \times N) \tilde{U}_{i}(\tilde{\alpha}, \tilde{\beta}, \tilde{D})=\left(\tilde{\alpha}_{1 i}, \tilde{\beta}_{1 i}, \tilde{D}_{1 i}, \ldots, \tilde{\alpha}_{N i}, \tilde{\beta}_{N i}, \tilde{D}_{N i}\right)^{T}$ is formed (expression (3)). The vector of unknown coordinates of the target at time $t_{i} \tilde{\xi}=(\tilde{x}(t), \tilde{y}(t), \tilde{z}(t))^{T}$ is the state vector. With a normal distribution law of estimates $\tilde{U}_{i}(\tilde{\alpha}, \tilde{\beta}, \tilde{D})=\left(\tilde{\alpha}_{1 i}, \tilde{\beta}_{1 i}, \tilde{D}_{1 i}, \ldots, \tilde{\alpha}_{N i}, \tilde{\beta}_{N i}, \tilde{D}_{N i}\right)^{T}$, the task of finding the vector of unknown coordinates of the target $\tilde{\xi}=(\tilde{x}(t), \tilde{y}(t), \tilde{z}(t))^{T}$ is solved by the maximum likelihood method, according to which the optimal estimate $\tilde{\xi}=(\tilde{x}(t), \tilde{y}(t), \tilde{z}(t))^{T}$ will be such that minimizes the quadratic form (5):

$$
L\left(\tilde{U}_{i}, \tilde{\xi}_{i}\right)=\text { const } \cdot \exp \left\{-\frac{1}{2}\left[\tilde{U}_{i}-U\left(\tilde{\xi}_{i}\right)\right]^{T} \cdot K_{U i}^{-1} \cdot\left[\tilde{U}_{i}-U\left(\tilde{\xi}_{i}\right)\right]\right\}
$$

where $K_{U i}$ is correlation matrix of errors (CME) of the $i$-th primary measurement of size $(3 \times N) \times(3 \times N)$; const is the factor before the exponent, which does not depend on $\tilde{\xi}_{i}$ and $\tilde{U}_{i}$.

The maximum of the likelihood function (5) is achieved when minimizing $\tilde{\xi}_{i}$ in the quadratic form $J\left(\tilde{\xi}_{i}\right)=\left[\tilde{U}_{i}-U\left(\tilde{\xi}_{i}\right)\right]^{T} \cdot K_{U i}^{-1} \cdot\left[\tilde{U}_{i}-U\left(\tilde{\xi}_{i}\right)\right]($ expression $(6))$ : 


$$
\tilde{J}=\min _{\xi_{i}}\left\{\left[\tilde{U}_{i}-U\left(\tilde{\xi}_{i}\right)\right]^{T} \cdot K_{U i}^{-1} \cdot\left[\tilde{U}_{i}-U\left(\tilde{\xi}_{i}\right)\right]\right\}
$$

The necessary condition for the minimum of the quadratic form $J\left(\tilde{\xi}_{i}\right)$ according to $\tilde{\xi}_{i}$ (expression (7)):

$$
\frac{\partial J\left(\tilde{\xi}_{i}\right)}{\partial \hat{\xi}_{i}}=\frac{\partial\left\{\left[\tilde{U}_{i}-U\left(\tilde{\xi}_{i}\right)\right]^{T} \cdot K_{U i}^{-1} \cdot\left[\tilde{U}_{i}-U\left(\tilde{\xi}_{i}\right)\right]\right\}}{\partial \hat{\xi}_{i}}=0
$$

is a system of equations for determining the estimate of the state vector $\tilde{\xi}_{i}=\left(\tilde{x}\left(t_{i}\right), \tilde{y}\left(t_{i}\right), \tilde{z}\left(t_{i}\right)\right)^{T}$. The equation of system (7) is nonlinear and to find the roots of system (7) it is advisable to use numerous (iterative) methods.

The calculation of the coordinates of the firing position in the CBA radars network consists in the fact that according to the marks of the observed it is necessary to determine the parameters of the target trajectory in three-dimensional space and to determine the coordinates of the firing position. The input data is the state vector (vector of coordinate estimates) of the target $\tilde{\xi}_{i}=\left(\tilde{x}\left(t_{i}\right), \tilde{y}\left(t_{i}\right), \tilde{z}\left(t_{i}\right)\right)^{T}$ at times $t_{i}$ and $K_{\xi_{i}}=\left(\left(B_{i}\right)^{T} K_{U i}^{-1} B_{i}\right)^{-1}$ is the estimate of the CME for determining the coordinates of the target $(i=1 \ldots I)$. The initial data $\tilde{x}_{A}, \tilde{y}_{A}$ - estimates of the coordinates of the firing position (provided that $\tilde{z}_{A}=0$ ). To calculate the trajectory of the target, it is necessary to numerically integrate the system of differential equations of external ballistics using a rather laborious finite difference method. To avoid these difficulties, let's use the results of [19-21]. This problem can be solved in two stages, thus reducing the dimension of the equations. First, it is necessary to determine the orientation of the firing plane relative to the horizontal plane, and then, in the found vertical plane, determine the parameters of the law of change in the height of the mine.

The sequence of actions of the method for determining the coordinates of the firing positions of roving mortars by the CBA radars network is shown in Fig. 1.

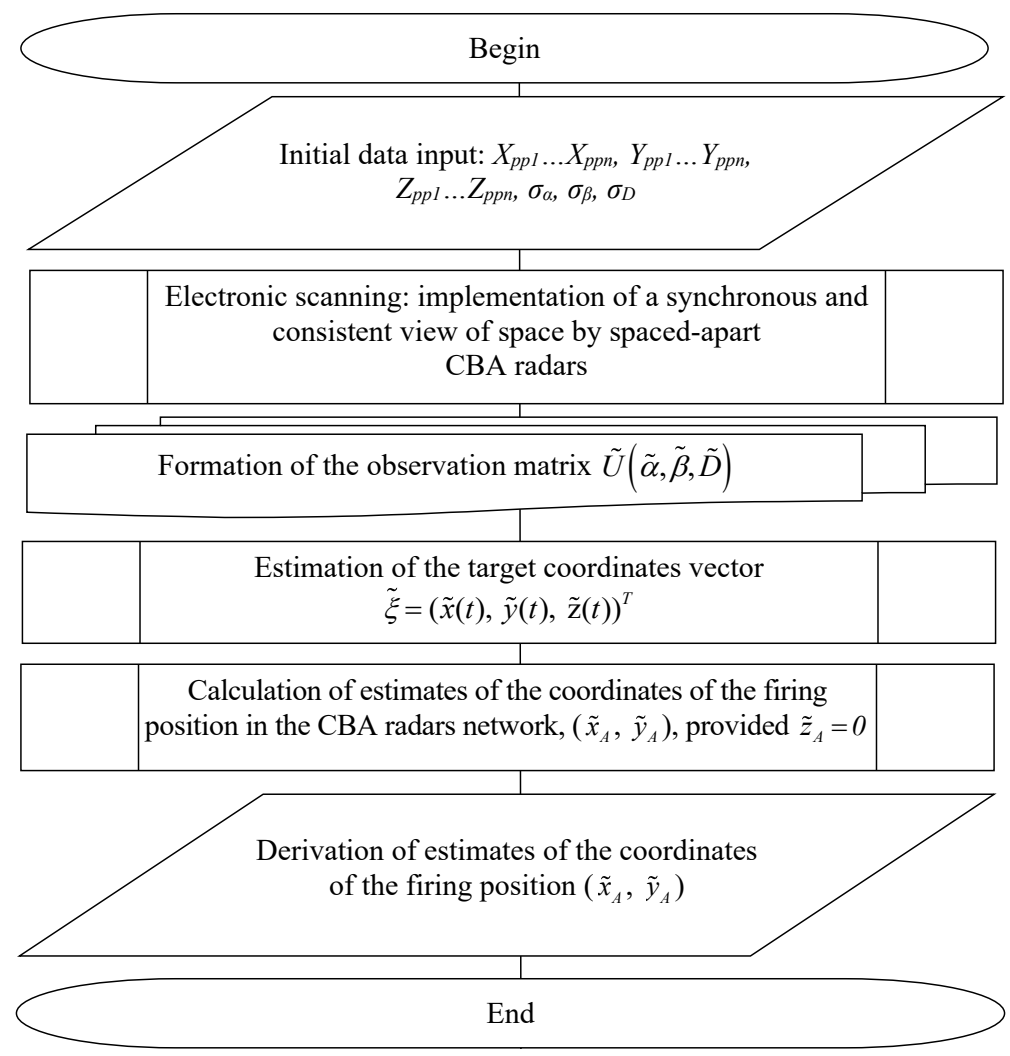

Fig. 1. Sequence of actions of the method for determining the coordinates of the firing positions of roving mortars by the CBA radars network 
The essence of the method is as follows:

1. Input of initial data, namely:

$-X_{p p 1} \ldots X_{p p N}, Y_{p p 1} \ldots Y_{p p N}, Z_{p p 1} \ldots Z_{p p N}$ - coordinates of the CBA radars included in the network;

$-\sigma_{\alpha}, \sigma_{\beta}, \sigma_{D}-$ measurement errors of azimuth, elevation and range (determined by the CBA radar tactical and technical characteristics (TTC)). It is believed that all CBA radars network are of the same type, have the same TTC and, accordingly, the same errors in the primary measurements of coordinates.

2. Electronic scanning of airspace: implementation of a space-synchronous and time-coordinated survey of space by spaced-apart CBA radars.

3. Formation of the observation matrix $\tilde{U}(\tilde{\alpha}, \tilde{\beta}, \tilde{D})$.

4. Estimation of the target coordinates vector $\tilde{\xi}=(\tilde{x}(t), \tilde{y}(t), \tilde{z}(t))^{T}$.

5. Calculation of estimates of the coordinates of the firing position in the CBA radars network $\left(\tilde{x}_{A}, \tilde{y}_{A}\right)$ (provided $\tilde{z}_{A}=0$ ).

To carry out a comparative assessment of the developed method for determining the coordinates of the firing positions of roving mortars and the known method of carrying out imitation modeling and assessing the quality of the method for determining the coordinates of the firing positions of roving mortars. Simulation statistical modeling is based on the statistical test method (Monte Carlo method).

Estimates of the accuracy of determining the coordinates of the firing position are shown in Fig. 2, 3. Graphs in Fig. 2, 3 are presented in the Cartesian coordinate system $(x, y)$. Fig. 2 shows the case of determining the coordinates of the firing position of one CBA radars. In Fig. 3 - determination of the coordinates of the firing position by a network of four CBA radars. CBA radars are indicated by triangles.
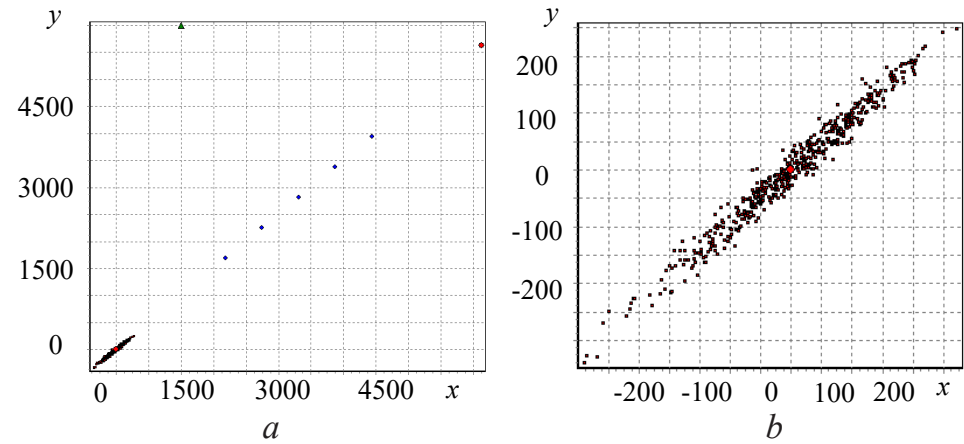

Fig. 2. Estimates of the accuracy of determining the coordinates of the firing position (one CBA radar): $a$ - the relative position of the firing position and the CBA radars; $b$ - scattering ellipses determining the coordinates of the firing position
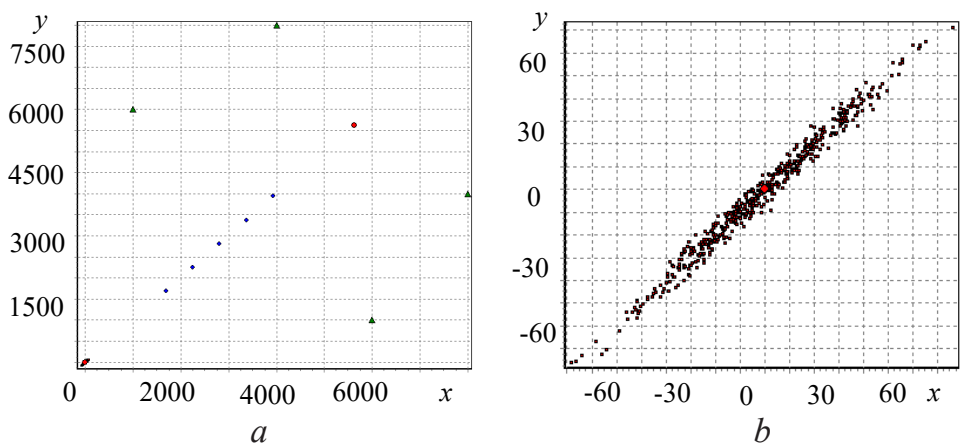

Fig. 3. Estimates of the accuracy of determining the coordinates of the firing position (a network of four CBA radars): $a$ - the relative position of the firing position and the CBA radars network; $b$ - scattering ellipses for determining the coordinates of the firing position 
Fig. 2, $\boldsymbol{a}, \mathbf{3}, \boldsymbol{a}$ show the relative position of the firing position and the CBA radars network. Fig. 2, b, 3, $\boldsymbol{b}$ show enlarged scattering ellipse for determining the coordinates of the firing position. Analysis of Fig. 2, 3, it was found that the use of the radars network makes it possible to increase the accuracy of determining the coordinates of the firing means on average from $23 \%$ to $71 \%$, depending on the number of CBA radars in the network.

In Fig. 4 shows the dependences of the errors in determining the coordinates of the firing position (semi-major $\sigma_{x}$ and semi-minor $\sigma_{y}$ axis of the scattering ellipse) on the number of CBA radars in the network $(N)$.
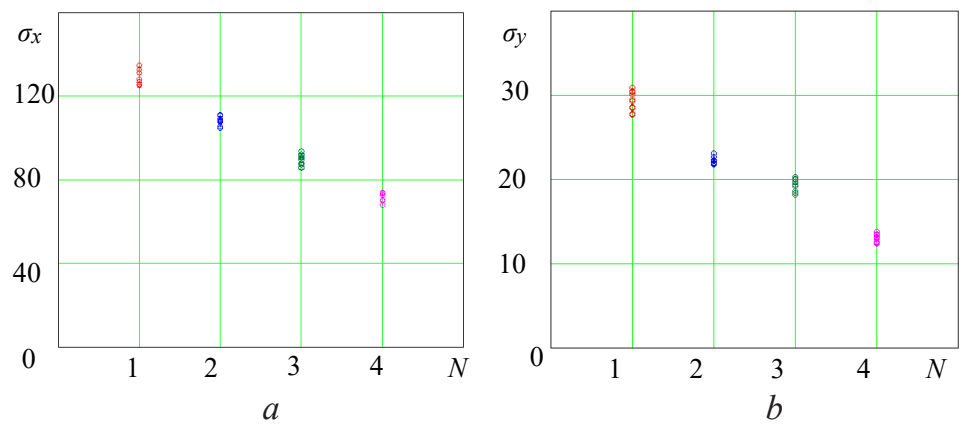

Fig. 4. Comparative assessment of the accuracy of determining the coordinates of the firing position: $a$-semi-major axis; $b$-semi-minor axis

Analysis of Fig. 4 it can be seen that the appropriate number of CBA radars elements in the network is three or four. A further increase in the number of CBA radars in the network does not lead to a significant increase in the accuracy of determining the coordinates of the firing position.

The increase in the accuracy of determining the coordinates of the firing positions of roving mortars is explained by the use of the CBA radars network.

A mathematical formulation of the problem of determining the coordinates of targets in the CBA radars network is formulated (expressions (1)-(7)). It has been established that the problem of estimating the coordinates of targets in the CBA radars network for an excessive number of estimates of primary coordinates should be considered as a statistical problem (expressions (5)-(7)).

The method for determining the coordinates of roving firing positions has been improved, in which, unlike the known ones, the coordinates of targets on the flight path are measured consistent with space and time and the information is processed by the CBA radars network (Fig. 1).

The developed simulation mathematical model for determining the coordinates of the firing positions of roving mortars by the CBA radars network.

Simulation modeling of the method for determining the coordinates of the firing positions of roving mortars by the CBA radars network was carried out.

It has been established that the use of a radar network makes it possible to increase the accuracy of determining the coordinates of the firing means on average from $23 \%$ to $71 \%$, depending on the number of CBA radars in the network (Fig. 2, 3).

It has been established that the appropriate number of CBA radars elements in the network is equal to three or four (Fig. 4).

A further increase in the number of CBA radars in the network does not lead to a significant increase in the accuracy of determining the coordinates of the firing positions of artillery and mortars (Fig. 4).

During the study, a single-purpose case was considered. In carrying out further research, it is necessary to develop a method for spatial separation of elements of a group of targets and interfering objects by the CBA radars network. At the same time, it is necessary to process large data arrays, which will require appropriate computing facilities. 


\section{Conclusions}

The method for determining the coordinates of the firing positions of roving mortars has been improved, in which, in contrast to the known ones, the coordinates of targets on the flight path are coordinated with space and time and the information is processed by the CBA radars network.

Simulation modeling of the method for determining the coordinates of the firing positions of roving mortars by the CBA radars network was carried out. It has been established that the use of a radar network makes it possible to increase the accuracy of determining the coordinates of the firing device on average from $23 \%$ to $71 \%$, depending on the number of CBA radars in the network.

It has been established that the appropriate number of CBA radars elements in the network is equal to three or four. A further increase in the number of CBA radars in the network does not lead to a significant increase in the accuracy of determining the coordinates of the firing positions of artillery and mortars.

\section{References}

[1] Counter-Rocket, Artillery, Mortar (C-RAM). Available at: https://missiledefenseadvocacy.org/defense-systems/counterrocket-artillery-mortar-c-ram/

[2] Meador, M. (2020). 5-5th ADA Soldiers certify joint C-RAM training. Available at: https://www.army.mil/article/232259/ 5_5th_ada_soldiers_certify_joint_c_ram_training

[3] Kisilyov, S., Piskunov, S., Filippenkov, A., Shevchenko, A. (2017). The counter-rockets, artillery and mortar concept as perspective field of development of air defense of ground forces. Systems of Arms and Military Equipment, 4 (52), 17-27.

[4] PdM Radars AN/TPQ-48. New Equipment Training. Introduction / Theory of Operations (2010).

[5] Humeur, R. (2017). Radar detection of artillery rockets: Report. Vorsvarshogskolan, 38.

[6] Richards, M. A., Scheer, J. A., Holm, W. A. (Eds.) (2010). Principles of Modern Radar: Basic principles. The Institution of Engineering and Technology, 924. doi: https://doi.org/10.1049/sbra021e

[7] Li, J., Stoica, P. (2008). MIMO radar signal processing. John Wiley \& Sons, Inc. doi: https://doi.org/10.1002/9780470391488

[8] Chernyak, V. S. (2012). Mnogopozitsionnye radiolokatsionnye sistemy na osnove MIMO RLS. Uspehi sovremennoy radioelektroniki, 8, 29-45.

[9] Ruban, I., Khudov, H., Lishchenko, V., Zvonko, A., Glukhov, S., Khizhnyak, I. et. al. (2020). The Calculating Effectiveness Increasing of Detecting Air Objects by Combining Surveillance Radars into The Coherent System. International Journal of Emerging Trends in Engineering Research, 8 (4), 1295-1301. doi: https://doi.org/10.30534/ijeter/2020/58842020

[10] Pyunninen, S. A. (2012). Method of detection of coordinates and motion parameters for nonlinear motion object using bearings-only information. Nauchniy zhurnal KubGAU. 78 (04).

[11] Lysiy, N. I., Gurman, I. V., Zvezhinskiy, S. S. (2013). Opredelenie mestopolozheniya obekta s ispol'zovaniem uluchshennoy trehtochechnoy passivnoy sistemy. Spetstehnika i svyaz', 2, 27-29.

[12] Potapova, T. P., Toporkov, N. V., Shabatura, Y. M. (2010). Algoritm opredeleniya koordinat istochnikov radioizlucheniya s letatel'nogo apparata na osnove fazovo-vremennoy signal'noy informatsii ot dvuh priemnyh moduley. Vestnik Moskovskogo gosudarstvennogo tehnicheskogo universiteta im. N. E. Baumana. Seriya «Priborostroenie», 1, 52-61.

[13] Il'yin, E. M., Klimov, A. E., Pashchin, N. S., Polubekhin, A. I., Cherevko, A. G., Shumskyi, V. N. (2015). Passive location systems. Perspectives and solutions. Vestnik SibGUTI, 2 (30), 7-20.

[14] Kondrat'ev, V. S., Kotov, A. F., Markov, L. N. (1986). Mnogopozitsionnye radiotehnicheskie sistemy. Moscow: Radio i svyaz', 264.

[15] Farina, A., Studer, F. A. (1986). Radar data processing. Volume 2 - Advanced topics and applications. Letchworth: Research studies press Ltd., 362.

[16] Hill, D., Galloway, P. (2008). Multi-Static Primary Surveillance Radar - An examination of Alternative Frequency Bands, 183. Available at: https://www.eurocontrol.int/sites/default/files/2019-05/surveillance-report-multi-static-primary-surveillanceradar-an-examination-of-altervative-frequency-bands-200807.pdf

[17] Kruglikov, S. V., Kruglikov, V. V. (2008). Sposob zaschity obektov ot udarov vysokotochnogo oruzhiya. Nauka i voyennaya bezopasnost', 1, 26-31.

[18] Skosyrev, V. N., Usachev, V. A. (2009). Tekhnicheskiye puti povysheniya energeticheskogo potentsiala radiolokatorov. Vestnik MGTU im. N. E. Baumana. Ser. «Priborostroyeniye», 78-89.

[19] Konosevich, B. I., Konosevich, Yu. B. (2015). Correctness of the modified point-mass model in the flight theory of the shell. Mekhanika tverdogo tela, 45, 11-25. 
[20] Konosevich, B., Konosevich, Y. (2017). Error estimate of the modified point-mass trajectory model of an artillery shell. Nonlinear Dynamics, 90 (1), 203-221. doi: https://doi.org/10.1007/s11071-017-3655-2

[21] Konosevich, B. I., Konosevich, Y. B. (2019). Comparison of two modified point-mass trajectory models of an artillery shell. Vestnik of Saint Petersburg University. Mathematics. Mechanics. Astronomy, 6 (64 (3)), 463-481. doi: https://doi.org/ 10.21638/11701/spbu01.2019.311

How to cite: Khudov, H., Yuzova, I., Lisohorskyi, B., Solomonenko, Y., Mykus, S., Irkha, A., Onishchuk, V., Sukonko, S., Semiv, G., Bondarenko, S. (2021). Development of methods for determining the coordinates of firing positions of roving mortars by a network of counter-battery radars. EUREKA: Physics and Engineering, 3, 140-150. doi: https://doi.org/10.21303/2504-5695.2021.001821 